\title{
Association Between Frontal Alpha Asymmetry With Cognitive Symptoms, Depression Severity, and Insomnia
}

\author{
Dae Yun Hwang', Yang Rae Kim², and Young-Min Park' \\ ${ }^{1}$ Department of Psychiatry, Ilsan Paik Hospital, Inje University College of Medicine, Goyang, Korea \\ ${ }^{2}$ Dr. Kim's Hue Psychiatric Office, Bucheon, Korea
}

\begin{abstract}
Objective: Previous studies have compared depressive episodes between bipolar disorder (BD) and major depressive disorder (MDD) using quantitative electroencephalogram (QEEG); however, there are no distinct discriminating feature between them. Here, we used QEEG to directly compare the alpha asymmetry and absolute power of each band between patients with BD and MDD. Methods: Fifty in-patients with major depressive episodes between 2019 and 2021 were retrospectively enrolled. Self-reported questionnaires including the Beck Depression Inventory (BDI), Korean version of the Childhood Trauma Questionnaire, and Adult Attention-Deficit/Hyperactivity Disorder Self Report Scale (ASRS) were used to evaluate the symptoms. The absolute power of QEEG delta, theta, alpha, beta, high beta waves, and the Z-scores of frontal alpha asymmetry were collected. A t-test and Pearson's correlation test were conducted using these data and based on these results, an analysis of covariance was conducted. Results: There were no significant differences between MDD and BD in QEEG power or alpha asymmetry. Patients with severe depression ( $B D I \geq 29)$ had higher alpha power at FP1 ( $p=0.037)$, FP2 ( $p=0.028), F 3(p=0.047)$, F4 ( $p=0.016)$, and higher right frontal alpha asymmetry at F3-F4 ( $p=0.039)$. Adult patients with features consistent with ADHD (ASRS $\geq 4)$ had higher right frontal alpha asymmetry at F3-F4 ( $p=0.046)$. Patients with insomnia had higher left frontal alpha asymmetry at F3-F4 ( $p=0.003)$. Conclusion: QEEG limited the differential diagnosis of MDD and BD. However, frontal alpha asymmetry did exist in depression and affected cognitive impairment, insomnia, and depression severity in particular. Future studies with improved methodologies are needed for a better comparison.
\end{abstract}

Keywords: Frontal alpha asymmetry; Quantitative electroencephalography; Depression; Adult ADHD; Insomnia

Received: November 19, 2021 Revised: December 8, 2021 Accepted: December 8, 2021

Corresponding author: Young-Min Park, MD, PhD, Department of Psychiatry, Ilsan Paik Hospital, Inje University College of Medicine, 170 Juhwa-ro, Ilsanseo-gu, Goyang 10380, Korea.

Tel: 82-31-910-7260, Fax: 82-31-910-7268, E-mail: medipark@hanmail.net

(a) This is an Open Access article distributed under the terms of the Creative Commons Attribution Non-Commercial License (https://creativecommons.org/licenses/bync/4.0) which permits unrestricted non-commercial use, distribution, and reproduction in any medium, provided the original work is properly cited.

\section{INTRODUCTION}

Depressive episodes in major depressive disorder (MDD) and bipolar disorder (BD) are clinically different. In BD, atypical features-including psychomotor retardation, hypersomnia, increased appetite, sensitivity to rejection (which is more frequent with an earlier age at onset), and past histories of postpartum depression or psychotic features-are more often compared to MDD [1]. However, though these results are based on comparisons between groups, applying these results to individual patients is difficult $[2,3]$. Consequently, MDD and BD have the same diagnostic criteria for depressive episodes. Thus, many researchers have investigated biological differences between bipolar de- pression and MDD. They have considered using biomarkers-in blood specimens-HPA axis, oxidative stress, or molecular biology to help address this [4-9]. Additionally, loudness dependence of auditory evoked potential (LDAEP) to test serotonergic transmission was used to study the differential response to the treatment $[10,11]$. However, all the aforementioned biomarkers had limitations, with inconsistent results. Therefore, we aimed to use quantified electroencephalogram (QEEG) to differentiate BD from MDD [4-9].

The QEEG, now popular, has been used to investigate the neurobiological characteristics of depression, with many studies showing an increase in left hemispheric frontal alpha power in depressed patients compared to healthy controls [12-15]. Since 
alpha power is inversely related to neuronal activity, it reflects reduced activity in the left frontal region in patients with depression [16-18]. Studies have attempted to use alpha asymmetry as a biomarker in patients with depression $[19,20]$; however, such studies on alpha asymmetry remain inconsistent. We postulated that the inconsistency of frontal alpha asymmetry was because of the heterogeneous nature of patients with depression and the inclusion of patients with bipolar depression within the sample. Additionally, since frontal alpha asymmetry is related to cognitive function and neuronal activity, and cognitive dysfunction is more prominent in bipolar depression than in MDD, we postulated that frontal alpha asymmetry would be more prominent in bipolar depression. Some studies attempted to compare depressive episodes in BD and MDD using QEEG; however, little is known so far, with no decisive hallmarks differentiating the two [21,22]. In this study, we intend to use QEEG to directly compare the alpha asymmetry and absolute power of each band between patients with BD and MDD.

\section{METHODS}

\section{Subjects and study design}

A total of 50 in-patients with major depressive episodes were retrospectively enrolled. This enrollment, based on medical records at the Ilsan Paik Hospital between 2019 and 2021, included patients, aged 17-71 years, diagnosed with either MDD $(n=29)$ or BD-type I, II, or other specified bipolar and related disorder $(n=21)$. Subjects who met the DSM-5 criteria for other major psychiatric disorders, such as schizophrenia or personality disorders, were excluded. The subjects were divided into MDD and $\mathrm{BD}$ groups according to DSM-5 criteria. QEEG recordings were collected, and self-reported questionnaires including Beck Depression Inventory (BDI), Korean version of Childhood Trauma Questionnaire (K-CTQ), Adult Attention-Deficit/Hyperactivity Disorder Self Report Scale (ASRS) were also used to collect clinical information. The BDI is a scale for measuring depression, which consists of 21 items with a three-point scale for each item [23]. We used the cutoff value for moderate depression, which was 20 [23]. Additionally, we also utilized the sleep-related item in BDI (item 16 - 0 : "I can sleep as well as usual," 1 : "I don't sleep as well as usual", 2: "I wake up 1-2 hours earlier than usual and find it hard to get back to sleep," 3: "I wake up several hours earlier than I used to and cannot get back to sleep") to find out factors that were associated with insomnia with a cutoff of 1 or higher [23]. In the KCTQ scale, all five subscales (sexual abuse, physical abuse, emotional abuse, physical neglect, and emotional neglect) were collected [24]. We used the cutoff values suggested by Bernstein et al. [24], the developer of CTQ. The ASRS was developed by Kessler et al. [25] and was used to screen adult ADHD; the first six items (Part A) were used in this study with a cutoff of 4 , which is consistent with adult ADHD.

The study protocol was approved by the ethics committee of Ilsan Paik Hospital before beginning the investigation (ISPAIK
2021-08-055). Informed consent was waived because of the retrospective nature of the study.

\section{QEEG and procedure}

The E-prime software (version 2, Psychology Software Tools, Pittsburgh, PA, USA) was used to synchronize QEEG recordings and onset of stimulus presentation recordings. A Neuroscan SynAmps amplifier (Compumedics USA, El Paso, TX, USA) with 64 $\mathrm{Ag}$-AgCl electrodes on a Quik-Cap was used to record the EEG in an extended 10-20 placement scheme. The specific protocol has been described elsewhere [26,27].

The absolute power of the delta $(1.0-4.0 \mathrm{~Hz})$, theta $(4.0-8.0 \mathrm{~Hz})$, alpha $(8.0-12.0 \mathrm{~Hz})$, beta $(12.0-25.0 \mathrm{~Hz})$, and high beta $(25.0-30.0$ $\mathrm{Hz}$ ) waves, and the $\mathrm{Z}$-scores of the alpha band wave asymmetry in the frontal lobe area were calculated with a confidence interval of $95 \%$. The nodes for the frontal lobe area were FP1, FP2, F3, F4, F7, and F8, and asymmetry for FP1-FP2, F3-F4, F7-F8 were calculated.

\section{Statistical analysis}

Participants were divided into two groups according to three categories: $\mathrm{MDD}$ vs. BD group, depression severity group (BDI $\geq 29$ vs. $\mathrm{BDI}<29$ ), ASRS positive $(\mathrm{ASRS} \geq 4)$ vs. negative $(\mathrm{ASRS}<4)$ group, based on the medical records and DSM-5. The groups were also divided by age (cutoff: 28 , median value) and sex. Tests such as t-test and Pearson's correlation test were conducted. Analysis of covariance (ANCOVA) was also performed; this was based on the t-test and Pearson's correlation test results, with age and sex as covariates and diagnoses (MDD vs. BD) as fixed factors. The ANCOVA tests for the BDI and ASRS were included as fixed factors. In the ANCOVA test for BDI scores, age, sex, and ASRS scores were added as covariates. Age, sex, and BDI scores were added as covariates in the ANCOVA test for ASRS scores.

All tests were two-tailed, and the cutoff p-value for statistical significance was set at $\mathrm{p}<0.05$. Statistical analysis was performed using SPSS software package (version 25, IBM Corp., Armonk, NY, USA).

\section{RESULTS}

Fifty patients were evaluated. The t-test was conducted by dividing them into two groups according to their clinical diagnoses (MDD and BD), age (cutoff: 28, median value), sex, BDI (cutoff: 20 [moderate] and 29 [severe]), and ASRS (cutoff: 4). The MDD group was significantly older $(\mathrm{p}=0.014)$, whereas the $\mathrm{BD}$ group had significantly higher physical neglect scores $(\mathrm{p}=0.044)$, a subscale of the K-CTQ. Older patients had higher right frontal alpha asymmetry at FP1-FP2 $(\mathrm{p}=0.044)$ and F7-F8 $(\mathrm{p}=0.021)$, with higher high beta power at FP1 $(\mathrm{p}=0.002), \mathrm{FP} 2(\mathrm{p}=0.005), \mathrm{F} 3(\mathrm{p}=$ $0.019)$, F7 ( $\mathrm{p}=0.025)$, and F8 $(\mathrm{p}=0.011)$. Women showed higher high beta power at FP1 ( $\mathrm{p}=0.031), \mathrm{FP} 2(\mathrm{p}=0.021), \mathrm{F} 3(\mathrm{p}=0.003)$, F4 ( $\mathrm{p}=0.041)$, and F7 ( $\mathrm{p}=0.029)$. Men had higher physical neglect scores $(\mathrm{p}=0.015)$. Patients with moderate depression (BDI scores 
$\geq 20)$ had higher total K-CTQ ( $\mathrm{p}=0.001)$ and emotional neglect $(\mathrm{p}=0.008)$ scores, and higher high beta and delta power at FP1 $(\mathrm{p}=0.017), \mathrm{F} 4(\mathrm{p}=0.002), \mathrm{F} 8(\mathrm{p}=0.013), \mathrm{FP} 2(\mathrm{p}=0.035), \mathrm{F} 3(\mathrm{p}=0.020)$, $\mathrm{F} 4(\mathrm{p}=0.013), \mathrm{F} 7(\mathrm{p}=0.040)$, and F8 ( $\mathrm{p}=0.040)$, respectively. Patients with severe depression (BDI scores $\geq 29$ ) showed higher emotional neglect $(\mathrm{p}=0.021)$ and higher right frontal alpha asymmetry at $\mathrm{F} 3-\mathrm{F} 4(\mathrm{p}=0.036)$.

In the Pearson correlation test, physical neglect correlated with $\mathrm{BDI}$ ( $\mathrm{p}=0.017, \mathrm{R}=0.352$ ), right frontal alpha asymmetry at $\mathrm{F} 3-\mathrm{F} 4$ ( $\mathrm{p}=0.044, \mathrm{R}=-0.325)$, delta band power at FP2 $(\mathrm{p}=0.049, \mathrm{R}=0.313)$, F3 ( $\mathrm{p}=0.025, \mathrm{R}=0.354), \mathrm{F} 4(\mathrm{p}=0.028, \mathrm{R}=0.348), \mathrm{F} 7(\mathrm{p}=0.023, \mathrm{R}=$

Table 1. Comparison of clinical variables among groups with MDD and $\mathrm{BD}$

\begin{tabular}{lcccc} 
Variable & $\begin{array}{c}\mathrm{MDD} \\
(\mathrm{n}=29)\end{array}$ & $\begin{array}{c}\mathrm{BD} \\
(\mathrm{n}=21)\end{array}$ & $\mathrm{F}$ & $\mathrm{p}$ \\
\hline Age $^{\dagger}$, years & $39.83 \pm 17.434$ & $28.29 \pm 13.058$ & 6.629 & 0.010 \\
$\mathrm{Sex}^{\ddagger}, \mathrm{M} / \mathrm{F}$ & $8 / 21$ & $6 / 15$ & $\mathrm{n} / \mathrm{a}$ & 0.593 \\
K-CTQ & $39.00 \pm 10.881$ & $45.19 \pm 16.321$ & 1.607 & 0.212 \\
Physical neglect & $6.85 \pm 2.461$ & $9.10 \pm 4.805$ & 5.230 & $0.027^{*}$ \\
ASRS & $3.04 \pm 2.010$ & $3.18 \pm 2.007$ & 0.045 & 0.833 \\
BDI & $30.74 \pm 10.697$ & $36.95 \pm 13.304$ & 3.145 & 0.083 \\
\hline
\end{tabular}

${ }^{*} \mathrm{p}<0.05$; ${ }^{\dagger}$-test; ${ }^{\prime}$ Fisher's exact test $\left(\chi^{2}=0.006\right)$. For the other variables, mean and standard deviation are based on t-test results, and $\mathrm{F}$ and $\mathrm{p}$ values are based on analysis of covariance results (age and sex are covariates in all variables except age and sex). For K-CTQ subscales and quantitative electroencephalogram data, only statistically significant results $(\mathrm{p}<0.05)$ were inserted in this table. MDD, major depressive disorder; $\mathrm{BD}$, bipolar disorder; K-CTQ, Korean version of the Childhood Trauma Questionnaire; ASRS, Adult Attention-Deficit/Hyperactivity Disorder Self-Report Scale; BDI, Beck Depression Inventory

Table 2. Comparison of clinical variables among groups divided by BDI scores

\begin{tabular}{lcccc}
\multicolumn{1}{c}{ Variable } & $\begin{array}{c}\mathrm{BDI} \geq 29 \\
(\mathrm{n}=27)\end{array}$ & $\begin{array}{c}\mathrm{BDI}<29 \\
(\mathrm{n}=20)\end{array}$ & $\mathrm{F}$ & $\mathrm{p}$ \\
\hline Age ${ }^{\dagger}$ years & $33.74 \pm 16.769$ & $35.70 \pm 17.036$ & 0.017 & 0.696 \\
Sex ${ }^{\ddagger}, \mathrm{M} / \mathrm{F}$ & $6 / 21$ & $6 / 14$ & $\mathrm{n} / \mathrm{a}$ & 0.392 \\
K-CTQ & $44.92 \pm 16.397$ & $37.00 \pm 7.685$ & 8.087 & $0.008^{*}$ \\
Emotional neglect & $12.38 \pm 6.217$ & $8.70 \pm 3.278$ & 12.661 & $0.001^{*}$ \\
Physical neglect & $8.50 \pm 4.159$ & $6.55 \pm 2.417$ & 4.823 & $0.035^{*}$ \\
ASRS & $3.30 \pm 2.285$ & $2.75 \pm 1.528$ & 0.949 & 0.337 \\
F3-F4 (Z-Score) & $-0.55 \pm 1.373$ & $0.40 \pm 1.389$ & 4.690 & $0.039^{*}$ \\
Alpha power (FP1) & $31.17 \pm 23.017$ & $22.83 \pm 21.657$ & 4.768 & $0.037^{*}$ \\
Alpha power (FP2) & $34.72 \pm 25.601$ & $24.73 \pm 24.694$ & 5.320 & $0.028^{*}$ \\
Alpha power (F3) & $36.12 \pm 25.473$ & $27.04 \pm 25.107$ & 4.296 & $0.047^{*}$ \\
Alpha power (F4) & $41.43 \pm 29.310$ & $27.39 \pm 27.173$ & 6.538 & $0.016^{*}$ \\
Alpha power (avg.) & $32.92 \pm 23.251$ & $24.60 \pm 24.464$ & 4.740 & $0.037^{*}$ \\
\hline
\end{tabular}

${ }^{*} \mathrm{p}<0.05$; †t-test; ${ }^{\ddagger}$ Fisher's exact test $\left(\chi^{2}=0.366\right)$. For the other variables, mean and standard deviation are based on $\mathrm{t}$-test results, and $\mathrm{F}$ and $\mathrm{p}$ values are based on analysis of covariance results (age, sex, and ASRS scores are covariates in all variables except age and sex). For K-CTQ subscales and quantitative electroencephalogram data, only statistically significant results $(\mathrm{p}<0.05)$ were inserted in this table. K-CTQ, Korean version of the Childhood Trauma Questionnaire; ASRS, Adult AttentionDeficit/Hyperactivity Disorder Self-Report Scale; BDI, Beck Depression Inventory; F3-F4, Z-score of frontal alpha asymmetry between each nodes
0.359), and $\mathrm{F} 8$ ( $\mathrm{p}=0.036, \mathrm{R}=0.332$ ). Total K-CTQ scores correlated with right frontal alpha asymmetry at $\mathrm{F} 3-\mathrm{F} 4(\mathrm{p}=0.012, \mathrm{R}=$ -0.396). Sexual abuse scores correlated with higher alpha band power at $\mathrm{F} 4(\mathrm{p}=0.020, \mathrm{R}=0.367)$. Age correlated with beta and high beta power at $\mathrm{FP} 1(\mathrm{p}=0.039, \mathrm{R}=0.316), \mathrm{F} 3(\mathrm{p}=0.049, \mathrm{R}=0.301)$ and FP1 ( $\mathrm{p}=0.000, \mathrm{R}=0.542), \mathrm{FP} 2(\mathrm{p}=0.004, \mathrm{R}=0.434), \mathrm{F} 3(\mathrm{p}=$ $0.008, \mathrm{R}=0.402), \mathrm{F} 7(\mathrm{p}=0.006, \mathrm{R}=0.416), \mathrm{F} 8(\mathrm{p}=0.015, \mathrm{R}=0.368)$, respectively.

ANCOVA tests were performed based on the Pearson and ttest results. In the ANCOVA results, patients with BD had significantly higher physical neglect scores $(\mathrm{p}=0.027)$ (Table 1$)$, but there were no other significant differences in band powers or frontal alpha asymmetry. Patients with severe depression (BDI score $\geq 29$ ) showed higher total K-CTQ ( $\mathrm{p}=0.008)$, emotional neglect $(\mathrm{p}=$ $0.001)$, and physical neglect $(\mathrm{p}=0.035)$ scores (Table 2$)$. These $\mathrm{pa}-$ tients also had higher alpha power at FP1 ( $\mathrm{p}=0.037), \mathrm{FP} 2(\mathrm{p}=0.028)$, $\mathrm{F} 3(\mathrm{p}=0.047)$, and F4 $(\mathrm{p}=0.016)$. Right frontal alpha asymmetry at $\mathrm{F} 3-\mathrm{F} 4$ was also prominent $(\mathrm{p}=0.039)$ (Table 2). Adult patients with symptoms consistent with ADHD (ASRS score $\geq 4$ ) had significantly higher right frontal alpha asymmetry at F3-F4 ( $\mathrm{p}=0.046)$ (Table 3). For insomnia, the ANCOVA test was performed with the BDI score of item No. 16 as a fixed factor (0 vs. 1 or higher) and age, sex, ASRS, and BDI as covariates. The results showed that patients with insomnia had higher physical abuse scores $(\mathrm{p}=0.012)$ and left frontal alpha asymmetry at $\mathrm{F} 3-\mathrm{F} 4(\mathrm{p}=0.003)$ (Figure 1).

\section{DISCUSSION}

The current study revealed no significant findings differentiating between patients with MDD and BD, except for physical neglect scores. However, when focusing on the depression severity rather than the diagnoses, patients with severe depression had significantly higher alpha power at FP1, FP2, F3, F4, and right frontal alpha asymmetry at F3-F4, with higher K-CTQ and physical and emotional neglect scores. Patients screened positive for ADHD showed significant right frontal alpha asymmetry at F3-

Table 3. Comparison of clinical variables among groups divided by ASRS scores

\begin{tabular}{lcccc} 
Variable & $\begin{array}{c}\text { ASRS } \geq 4 \\
(\mathrm{n}=17)\end{array}$ & $\begin{array}{c}\text { ASRS }<4 \\
(\mathrm{n}=24)\end{array}$ & $\mathrm{F}$ & $\mathrm{p}$ \\
Age $^{\dagger}$, years & $27.29 \pm 9.885$ & $34.71 \pm 17.938$ & 15.889 & 0.99 \\
Sex $^{\ddagger}, \mathrm{M} / \mathrm{F}$ & $7 / 10$ & $5 / 19$ & $\mathrm{n} / \mathrm{a}$ & 0.144 \\
K-CTQ & $38.69 \pm 15.014$ & $41.27 \pm 11.601$ & 2.065 & 0.160 \\
BDI & $38.06 \pm 13.801$ & $30.52 \pm 11.212$ & 3.339 & 0.076 \\
F3-F4 (Z-Score) & $-0.91 \pm 1.231$ & $0.32 \pm 1.468$ & 4.336 & $0.046^{*}$ \\
\hline
\end{tabular}

${ }^{*} \mathrm{p}<0.05$; ${ }^{\mathrm{t}}$-test; ${ }^{\ddagger}$ Fisher’s exact test $\left(\chi^{2}=1.989\right)$. For the other variables, mean and standard deviation are based on $\mathrm{t}$-test results, and $\mathrm{F}$ and $\mathrm{p}$ values are based on analysis of covariance results (age, sex, and depression severity are covariates in all variables except age and sex). For K-CTQ subscales and quantitative electroencephalogram data, only statistically significant results $(\mathrm{p}<0.05)$ were inserted in this table. K-CTQ, Korean version of the Childhood Trauma Questionnaire; ASRS, Adult Attention-Deficit/Hyperactivity Disorder Self-Report Scale; BDI, Beck Depression Inventory; F3-F4, Z-score of frontal alpha asymmetry between each nodes 


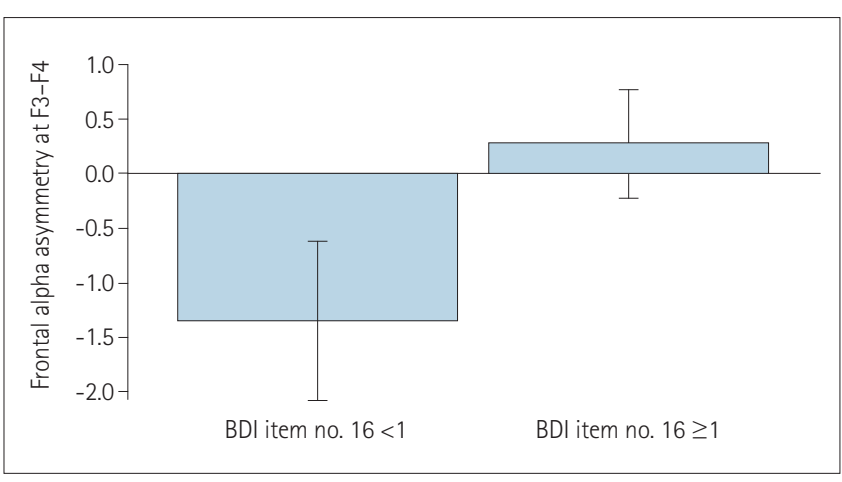

Figure 1. Comparison of frontal alpha asymmetry at F3-F4 among groups divided by insomnia $(p=0.003)$. BDI, Beck Depression Inventory.

F4. Patients with insomnia had significantly higher physical abuse scores and left frontal alpha asymmetry at F3-F4. In short, frontal alpha asymmetry was associated with cognitive symptoms, depression severity, and insomnia.

There have been few previous attempts to differentiate between $\mathrm{MDD}$ and $\mathrm{BD}$ patients using QEEG. Some investigators have attempted to differentiate MDD, BD patients, and healthy controls using EEG activity, and found that patients with MDD had significantly higher left frontal asymmetry compared to healthy controls; however, there were no significant differences in frontal alpha asymmetry in other comparisons [28]. However, other investigators have reported no differences in frontal asymmetry between MDD and BD [29]. Although our results did not fully discriminate between patients with $\mathrm{MDD}$ and $\mathrm{BD}$, there were significant results when focusing on depression severity. One study revealed that alpha power increased, with higher left frontal alpha asymmetry in patients with MDD compared with healthy controls [30]. The same study also found that compared to patients with MDD without suicidal ideation, MDD patients with suicidal ideation had significantly lower left frontal alpha asymmetry, with reduced alpha power in the left frontal region [30]. Since suicidal behavior is a strong indicator of severe depression, this suggests that alpha asymmetry can indeed be meaningful in assessing depression severity. Conversely, our results showed right frontal alpha asymmetry in patients with severe depression. This is contradictory not only to the findings described above, but also to the conventional hypothesis that there is left frontal alpha asymmetry in patients with depression. This may be due to the heterogeneous nature of depression and inconsistent findings regarding the aforementioned hypothesis. However, because our findings are statistically significant, other factors, such as cognitive impairment, might have played a role in this result.

Several previous studies have shown a relationship between frontal alpha asymmetry and adult ADHD. A recent study revealed that ADHD symptoms meant significantly stronger relative right frontal alpha power in adults with ADHD [31]. In another study, patients with ADHD showed significantly stronger anterior cortical activity in the left hemisphere in adults [32]. An additional study also revealed the relationship between ADHD and frontal alpha asymmetry, suggesting that ADHD severity could be predicted by right frontal alpha asymmetry [33]. These findings were consistent with our results. However, one study reported left frontal alpha asymmetry in patients with $\mathrm{ADHD}$, which is inconsistent with the findings discussed above [34]. Some studies have pointed out that worse ADHD symptoms meant significantly suppressed cognitive function $[35,36]$. Considering this, we suggest that a higher ASRS score can be interpreted as poorer cognition, and it is possible that cognitive impairment itself can contribute to frontal alpha asymmetry. Altogether, ADHD symptoms and cognitive impairment can both contribute to frontal alpha asymmetry.

Our results showed left alpha asymmetry in patients with insomnia. Attempts have been made to correlate frontal alpha asymmetry with sleep. One study found that sleep-deprivation induced significantly greater right-side alpha activation in the frontal region [37]. Another study revealed that frontal alpha asymmetry during wakefulness positively correlated with frontal alpha asymmetry during REM sleep [38]. Researchers have also found that dream anger can be predicted by right frontal alpha asymmetry during REM sleep [38]. Emotional dysregulation can contribute to insomnia, and lack of sleep itself can also affect left frontal alpha asymmetry [39]. Thus, the relationship between frontal alpha asymmetry and insomnia remains unclear. That may be due to the heterogeneity and confounders of depression that can affect QEEG results, such as education, medication, and handedness, could have accounted for these findings.

The current study has several limitations. This was a retrospective study, and the healthy control group was absent. Additionally, control for other confounders such as education, medication, and handedness was not considered. Patients with $\mathrm{BD}$ were mostly had bipolar II, with only two and one patients with bipolar I, and specified bipolar and related disorder, respectively. Therefore, our findings mostly reflected the aspects of bipolar II patients, rather than other types of bipolar disorders. Future research is required to address this issue. Despite this, the current study revealed that frontal alpha asymmetry did exist in depression and is affected by ADHD symptoms such as cognitive impairment, insomnia, and depression severity in particular. However, the current study suggests that QEEG, including frontal alpha asymmetry, has limitations in the differential diagnosis of MDD and BD. In future studies, methodological improvement with larger clinical samples or further control for confounders such as education, medication, and handedness would be necessary for better comparison.

\section{Acknowledgments}

This study was supported by 'Development of a biosensor-based mental health diagnosis and XR digital treatment platform for medically vulnerable areas and the underprivileged' funded by the Ministry of Science and Technology Information and Communication. 


\section{Conflicts of Interest}

The authors have no potential conflicts of interest to disclose.

\section{Author Contributions}

Conceptualization: Young-Min Park. Data curation: Dae Yun Hwang. Formal analysis: Dae Yun Hwang, Young-Min Park. Funding acquisition: Young-Min Park. Investigation: Dae Yun Hwang, Young-Min Park. Methodology: all authors. Project administration: Young-Min Park. Resources: Young-Min Park. Software: Young-Min Park. Supervision: Young-Min Park. Validation: all authors. Visualization: all authors. Writing - original draft: Dae Yun Hwang, Young-Min Park. Writing-review \& editing: Yang Rae Kim, Young-Min Park.

\section{ORCID iDs}

Dae Yun Hwang (1)

https://orcid.org/0000-0003-1764-8404

Young-Min Park (1)

https://orcid.org/0000-0002-4993-1426

\section{REFERENCES}

1. Łojko D, Buzuk G, Owecki M, Ruchała M, Rybakowski JK. Atypical features in depression: association with obesity and bipolar disorder. J Affect Disord 2015;185:76-80.

2. Cuellar AK, Johnson SL, Winters R. Distinctions between bipolar and unipolar depression. Clin Psychol Rev 2005;25:307-339.

3. Hirschfeld RM. Differential diagnosis of bipolar disorder and major depressive disorder. J Affect Disord 2014;169 Suppl 1:S12-S16.

4. Black CN, Bot M, Scheffer PG, Cuijpers P, Penninx BW. Is depression associated with increased oxidative stress? A systematic review and meta-analysis. Psychoneuroendocrinology 2015;51:164-175.

5. Gururajan A, Clarke G, Dinan TG, Cryan JF. Molecular biomarkers of depression. Neurosci Biobehav Rev 2016;64:101-133.

6. Juruena MF, Bocharova M, Agustini B, Young AH. Atypical depression and non-atypical depression: is HPA axis function a biomarker? A systematic review. J Affect Disord 2018;233:45-67.

7. Mora C, Zonca V, Riva MA, Cattaneo A. Blood biomarkers and treatment response in major depression. Expert Rev Mol Diagn 2018;18:513-529.

8. Thase ME. Using biomarkers to predict treatment response in major depressive disorder: evidence from past and present studies. Dialogues Clin Neurosci 2014;16:539-544.

9. Uddin M. Blood-based biomarkers in depression: emerging themes in clinical research. Mol Diagn Ther 2014;18:469-482.

10. Lee BH, Park YM, Lee SH, Shim M. Prediction of long-term treatment response to selective serotonin reuptake inhibitors (SSRIs) using scalp and source loudness dependence of auditory evoked potentials (LDAEP) analysis in patients with major depressive disorder. Int J Mol Sci 2015;16:62516265.

11. Park YM. Relationship between serotonergic dysfunction based on loudness dependence of auditory-evoked potentials and suicide in patients with major depressive disorder. Psychiatry Investig 2015;12:421-424.

12. Davidson RJ. Anterior cerebral asymmetry and the nature of emotion. Brain Cogn 1992;20:125-151.

13. Davidson RJ, Schaffer CE, Saron C. Effects of lateralized presentations of faces on self-reports of emotion and EEG asymmetry in depressed and nondepressed subjects. Psychophysiology 1985;22:353-364.

14. Henriques JB, Davidson RJ. Left frontal hypoactivation in depression. J Abnorm Psychol 1991;100:535-545.

15. Tucker DM, Stenslie CE, Roth RS, Shearer SL. Right frontal lobe activation and right hemisphere performance. Decrement during a depressed mood. Arch Gen Psychiatry 1981;38:169-174.

16. Pfurtscheller G, Stancák A Jr, Neuper C. Event-related synchronization
(ERS) in the alpha band--an electrophysiological correlate of cortical idling: a review. Int J Psychophysiol 1996;24:39-46.

17. Pizzagalli DA, Sherwood RJ, Henriques JB, Davidson RJ. Frontal brain asymmetry and reward responsiveness: a source-localization study. Psychol Sci 2005;16:805-813.

18. Goldman RI, Stern JM, Engel J Jr, Cohen MS. Simultaneous EEG and fMRI of the alpha rhythm. Neuroreport 2002;13:2487-2492.

19. Debener S, Beauducel A, Nessler D, Brocke B, Heilemann H, Kayser J. Is resting anterior EEG alpha asymmetry a trait marker for depression? Findings for healthy adults and clinically depressed patients. Neuropsychobiology 2000;41:31-37.

20. Dharmadhikari AS, Jaiswal SV, Tandle AL, Sinha D, Jog N. Study of frontal alpha asymmetry in mild depression: a potential biomarker or not? J Neurosci Rural Pract 2019;10:250-255.

21. Lieber AL, Newbury ND. Diagnosis and subtyping of depressive disorders by quantitative electroencephalography: III. Discriminating unipolar from bipolar depression. Hillside J Clin Psychiatry 1988;10:165-172.

22. Kim JS, Oh S, Jeon HJ, Hong KS, Baek JH. Resting-state alpha and gamma activity in affective disorder with ADHD symptoms: comparison between bipolar disorder and major depressive disorder. Int J Psychophysiol 2019; 143:57-63.

23. Beck AT, Ward CH, Mendelson M, Mock J, Erbaugh J. An inventory for measuring depression. Arch Gen Psychiatry 1961;4:561-571.

24. Bernstein DP, Stein JA, Newcomb MD, Walker E, Pogge D, Ahluvalia T, et al. Development and validation of a brief screening version of the childhood trauma questionnaire. Child Abuse Negl 2003;27:169-190.

25. Kessler RC, Adler L, Ames M, Demler O, Faraone S, Hiripi E, et al. The World Health Organization adult ADHD self-report scale (ASRS): a short screening scale for use in the general population. Psychol Med 2005;35:245256.

26. Jin MJ, Jung W, Hyun MH, Lee SH. Effect of behavioral inhibition system and childhood emotional neglect on serotonergic activity, negative affect, and rejection sensitivity in non-clinical adults. PLoS One 2018;13:e0207746.

27. Kim YR, Park YM. Mismatch negativity and loudness dependence of auditory evoked potentials among patients with major depressive disorder, bipolar II disorder, and bipolar I disorder. Brain Sci 2020;10:789.

28. Koller-Schlaud K, Ströhle A, Bärwolf E, Behr J, Rentzsch J. EEG frontal asymmetry and theta power in unipolar and bipolar depression. J Affect Disord 2020;276:501-510.

29. Tas C, Cebi M, Tan O, Hızlı-Sayar G, Tarhan N, Brown EC. EEG power, cordance and coherence differences between unipolar and bipolar depression. J Affect Disord 2015;172:184-190.

30. Roh SC, Kim JS, Kim S, Kim Y, Lee SH. Frontal alpha asymmetry moderated by suicidal ideation in patients with major depressive disorder: a comparison with healthy individuals. Clin Psychopharmacol Neurosci 2020;18: 58-66.

31. Keune PM, Wiedemann E, Schneidt A, Schönenberg M. Frontal brain asymmetry in adult attention-deficit/hyperactivity disorder (ADHD): extending the motivational dysfunction hypothesis. Clin Neurophysiol 2015;126:711720.

32. Keune PM, Schönenberg M, Wyckoff S, Mayer K, Riemann S, Hautzinger $\mathrm{M}$, et al. Frontal alpha-asymmetry in adults with attention deficit hyperactivity disorder: replication and specification. Biol Psychol 2011;87:306-310.

33. Alperin BR, Smith CJ, Gustafsson HC, Figuracion MT, Karalunas SL. The relationship between alpha asymmetry and ADHD depends on negative affect level and parenting practices. J Psychiatr Res 2019;116:138-146.

34. Jaworska N, Berrigan L, Ahmed AG, Gray J, Korovessis A, Fisher DJ, et al The resting electrophysiological profile in adults with ADHD and comorbid dysfunctional anger: a pilot study. Clin EEG Neurosci 2013;44:95-104.

35. Das D, Cherbuin N, Anstey KJ, Easteal S. ADHD symptoms and cognitive abilities in the midlife cohort of the PATH through life study. J Atten Disord 2015;19:414-424.

36. Das D, Cherbuin N, Easteal S, Anstey KJ. Attention deficit/hyperactivity disorder symptoms and cognitive abilities in the late-life cohort of the PATH through life study. PLoS One 2014;9:e86552.

37. Ferreira C, Deslandes A, Moraes H, Cagy M, Basile LF, Piedade R, et al. The relation between EEG prefrontal asymmetry and subjective feelings of mood following 24 hours of sleep deprivation. Arq Neuropsiquiatr 2006;64:382387. 
38. Sikka P, Revonsuo A, Noreika V, Valli K. EEG frontal alpha asymmetry and dream affect: alpha oscillations over the right frontal cortex during rem sleep and presleep wakefulness predict anger in REM sleep dreams. J Neu- rosci 2019;39:4775-4784

39. Coan JA, Allen JJ. Frontal EEG asymmetry as a moderator and mediator of emotion. Biol Psychol 2004;67:7-49. 